\title{
A new numerical framework to investigate variations of weathering- tracing isotope systems in the sedimentary record
}

\author{
MARKUS ADLOFF ${ }^{1,2}$, SARAH GREENE ${ }^{1}$, FANNY \\ MONTEIRO $^{2}$ AND ANDY RIDGEWELL ${ }^{3}$
}

${ }^{1}$ University of Birmingham

${ }^{2}$ University of Bristol

${ }^{3}$ University of California - Riverside

Presenting Author: m.adloff@bham.ac.uk

The isotopic composition of ancient marine sediments can be used to trace continental and volcanic additions of $\mathrm{Sr}, \mathrm{Os}, \mathrm{Li}$ and $\mathrm{Ca}$ to the ocean, and therefore to investigate the coupling between land, ocean and lithosphere in past Earth system states and during episodes of global environmental change. Isotope mixing models for metal and carbon cycles provide interpretive tools for preserved isotopic changes in individual proxy systems, but their different degrees of complexity and underlying assumptions complicate combined interpretations. Such combined numerical analysis was hitherto only possible for global isotopic changes on geologic timescales. Here, we present our implementation of isotope-enabled $\mathrm{Sr}, \mathrm{Os}, \mathrm{Li}$ and $\mathrm{Ca}$ cycling in cGENIE, making it the first numerical tool which can simulate and assimilate local records of weathering and climate proxies on timescales of centuries to millions of years in a $3 \mathrm{D}$ Earth system. In CGENIE, these metals and carbon interact and react to external forcing in one consistent framework, enabling the first simulations of the simultaneous effects of spatially diverse Earth system changes on these elements and their isotopes, and their transient responses to environmental change. In the presentation, I will give a brief overview of the metal cycles and processes added to cGENIE and show how this new model framework can be used to constrain the scale of lithological processes in the past. 